EXTENDED REPORT

\title{
Contribution to comprehension of image formation in confocal microscopy of cornea with Rostock cornea module
}

\author{
R Bochert, A Zhivov, R Kraak, J Stave, R F Guthoff
}

Br J Ophthalmol 2005;89:1351-1355. doi: 10.1136/bjo.2004.063743

\begin{abstract}
See end of article for authors' affiliations

.....................

Correspondence to: Dr Ralf Bochert Robert Kraak, Universität Rostock, Universitätsaugenklinik, Doberaner Strasse 140, D18055 Rostock, Germany; ralf.bo@t-online.de

Accepted for publication 1 February 2005
\end{abstract}

\begin{abstract}
Aim: To investigate the influence of refractive index of aqueous humour on imaging of corneal endothelium in confocal microscopy. To clarify the phenomenon of dark endothelial and bright epithelial cell membranes in confocal images of corneas.

Methods: Use of a novel digital confocal laser scanning microscope, a combination of the Heidelberg retina tomograph (HRT II) and the Rostock cornea module. Exchange of aqueous humour solution from domestic pigs against glycerol/water solutions (refractive indices $\eta=1.337-1.47$ ). Transelectron microscopy of endothelial and epithelial cell morphology.

Results: Under the terms of variable refractive indices no differences were observed for general imaging of endothelium. Bright cells were bordered by dark cell membranes in all experiments. Electron microscopy of endothelium and epithelium revealed differences in intracellular and cell membrane structure of both cell types.

Conclusion: Source of specific confocal optical behaviour of endothelium does not come from interface conditions to aqueous humour, but may result from intracellular variations and ultrastructure of cell membranes.
\end{abstract}

imaging of cells in the epithelium and the endothelium. All cells of the five to six cellular layers of the epithelium are visible with bright cell borders and dark homogeneous cytoplasm. In contrast, the one layer of endothelial cells at the border of the anterior chamber is pictured with dark cell borders and bright cytoplasm. ${ }^{6}$

This study describes to what extent the refractive index, $\eta$, of aqueous humour and the overlying cell layers affects the image formation of the corneal endothelium. A simple stacking experiment of two corneas is used to describe potential influence of focal depth within tissue on backscattered light intensity. Electron microscopic investigation magnifies morphological structures of different cell types for comparison.

\section{MATERIAL AND METHODS}

\section{Confocal microscopy}

A novel digital confocal laser scanning microscope (LSM), a combination of the Heidelberg retina tomograph (HRT II) and the Rostock cornea module (Heidelberg Engineering GmbH) was used.' The LSM has a computer controlled hydraulic linear scanning device (Nikon hydraulic manipulator, Tokyo, Japan) and a water contact objective (Zeiss, $63 \times / \mathrm{NA} 0.95 \mathrm{~W})$. A diode laser emits a beam of $670 \mathrm{~nm}$ wavelength. One enucleated eye ball of a domestic pig was fixed in front of the LSM objective. The eye was in close contact with a Plexiglas disc, which covered the LSM objective and allowed the objective to move in z-axis while the eye rested in close contact to the Plexiglas surface. A drop of carbomer gel (Visidic, Dr Mann Pharma, Berlin, Germany) served as the coupling medium. A feature of the LSM is the ability to perform hydraulic z-scans, beginning from the contact area of the Plexiglas disc/surface of the cornea to a maximum of $2000 \mu \mathrm{m}$ depth. The actual focus depth within the cornea is displayed. The saving of images $(300 \times 300 \mu \mathrm{m}$ corneal area) of all layers from the superficial epithelium to

Abbreviations: FDTD, finite difference time domain; LSM, laser scanning microscope 
endothelium of the corneas is possible. A digital colour photo camera at one side can be use to assess and document the alignment of eye within laser beam.

All image contrast arises from the interaction of the incident light beam with the object. The focus depth of the LSM is $10 \mu \mathrm{m}$. This is the volume (voxel) which is imaged by the confocal microscope to form an image pixel. All investigations were performed in the central region of the cornea.

Investigation objects were eyeballs from the domestic pig (Sus scrofa $f$ domestica). Domestic pigs were about 6 months old and slaughtered in the research institute for the biology of farm animals (Dummerstorf, Germany).

To clarify the extent of several variables on image formation different study designs were used. Changes of conditions at the interface endothelium/aqueous humour describe the influence of refractive index. The amount of weakening on laser beam through tissue layers overlying the focal plane describes an experiment of two corneas. Divergent from numerical measurement of intrinsic light scattering intensity as a useful objective parameter, only the clear visible scattering contrast of cytoplasm and cell membranes was used for evaluation. Additionally, electron microscopic investigation magnifies morphological structures of different cell types for comparison.

\section{Experiment 1}

To test the influence of refractive index, $\eta$, on imaging of the endothelial cells a dilution series of glycerol (Merck, Germany) and distilled water was made (table 1). The refractive index value of each solution was measured by means of an Abbe refractometer model G (C Zeiss Jena, Germany).

Altogether, 18 eyes (three per set) were used. Eyeballs of domestic pigs were prepared for investigation as followed. The cornea was punctured peripherally with a small needle and the aqueous humour was extracted with a syringe. The anterior chamber was subsequent filled by injecting one of the glycerol/water solutions. This procedure took 2 minutes and endothelium was then imaged by the confocal microscope.

\section{Experiment 2}

To test the amount of contrast degradation of the cell layers overlying the endothelium, two eyeballs were used simultaneously. A portion of the central cornea with a diameter of about $6 \mathrm{~mm}$ was cut from one eyeball (specimen 1). This piece of cornea was placed on top with the endothelium side of the epithelium of specimen 2. A drop of carbomer gel (Visidic, Dr Mann Pharma, Berlin, Germany) served as coupling medium. This double cornea was then imaged by the confocal microscope.

\section{Trans-electron microscopy}

Corneas of domestic pigs were prepared for trans-electron microscopic investigation. Specimens were fixed with $4 \%$

Table 1 Refractive index values of a dilution series of glycerol and distilled water

\begin{tabular}{ll}
\hline Percentage glycerol/distilled water & $\eta \mathbf{D}(\mathbf{2 0})$ \\
\hline $5 \%$ & 1.337 \\
$20 \%$ & 1.355 \\
$40 \%$ & 1.381 \\
$60 \%$ & 1.411 \\
$80 \%$ & 1.440 \\
$100 \%$ & 1.470 \\
\hline
\end{tabular}

glutaraldehyde in $0.2 \mathrm{M}$ Sörrensen's phosphate buffer ( $\mathrm{pH} 7.4$ ) for 2 hours and then post-fixed with $1 \%$ osmium tetroxide for 1 hour. After dehydration in acetone, the corneas were embedded in araldite. Sections were contrasted by immersion in saturated uranyl acetate solution in 50\% methanol for 30 minutes and Reynolds lead citrate solution for 4 minutes. Ultrathin sections parallel to corneal surface of the epithelium and the endothelium were examined under a Zeiss EM 902A electron microscope.

\section{RESULTS}

\section{Experiment 1}

The exchange of aqueous humour against different solutions of glycerol/water revealed relatively clear imaging of the endothelium. Under refractive index values from 1.337 to 1.47, no differences in endothelium morphology were seen. In all investigations the cells were revealed with dark cell borders and bright cytoplasms (fig 1). Additionally, dark spots in the cytoplasm at $20 \%, 60 \%$, and $100 \%$ glycerol/water solution were visible, denoting the nuclei of each cell.

\section{Experiment 2}

Confocal image of the corneal endothelium of specimen 1 showed normal appearance. Dark cell borders, bright cytoplasm, and dark cell nuclei were visible. The contrast of epithelial cells of a second underlying cornea of specimen 2 was noticeably reduced. Nevertheless, the normal picture was recognisable. Superficial and wing epithelial cells showed bright cell membranes, bright nuclei, and dark cytoplasm (fig 2).

\section{Trans-electron microscopy}

Electron microscopic investigations showed the polygonal shape of the wing cells of the corneal epithelium (fig 3A). The cell membranes of this layer show deep interdigitations (fig 3B). Numerous desmosomal junctions join these cells to each other. The cytoplasm of the wing cells contained only moderate numbers of small thin mitochondria. Vesicles of different size were a prominent finding in the cytoplasm. The oval nuclei contained dense packages of heterochromatin. The lateral cell membranes between adjacent endothelial cells appeared smooth and showed no complex interdigitations. Desmosomes were lacking (fig 4). With respect to the anterior-posterior axis of the cornea, the lateral cell membranes of the endothelium have an oblique and tortuous course. The endothelial cell cytoplasm is rich in organelles. The numerous mitochondria are elongated and their cristae are oriented longitudinally. Both rough and smooth surfaced endoplasmic reticulum were present. The oval nuclei showed a homogeneous appearance without dense heterochromatin.

\section{DISCUSSION}

The source of contrast in backscattered confocal microscopic images of living species is based upon a variety of physical processes and species specific morphological, anatomical, and biochemical conditions. The incident light beam interacts in multiple ways with structures of the object in and above the focus plane of the objective. ${ }^{6}$ The specific source of contrast in backscattering confocal microscopy of detailed cellular structures, such as cell membranes, organelles, or cytoplasm within a tissue layer of a living object—for instance, the cornea, is not measurable directly.

Scattering in tissue arises from local changes in the index of refraction between cell components and other small tissue structures, and the scale over which these changes occur varies from tissue to tissue. ${ }^{10}$ The amount of scattered light depends on changes in local absorption and transmission. The light could be further affected by these different processes again, while the backscattered beam passes 

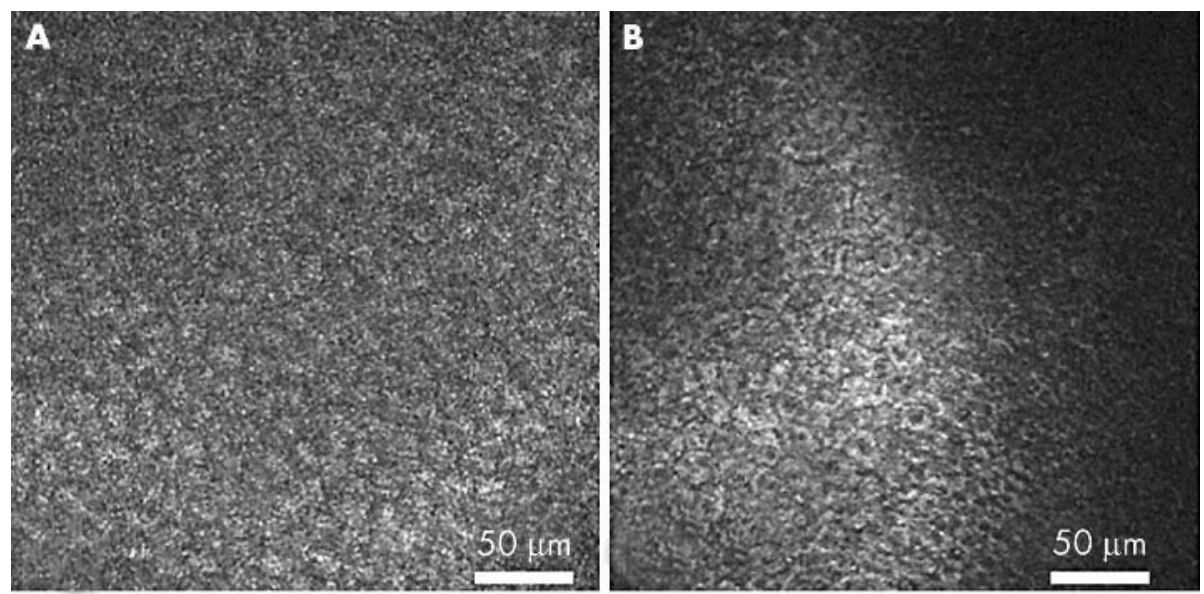

Figure 1 Confocal images of endothelium of domestic pig eyes. The anterior chamber was filled with glycerol/water solution of (A) $5 \%$ ( $\mathrm{D} D=1.337),(\mathrm{B}) 20 \%(\eta \mathrm{D}=1.355),(\mathrm{C})$ $60 \%(\eta D=1.411)$, and (D) $100 \%$ $(\eta \mathrm{D}=1.47)$. Magnification $\times 240$.
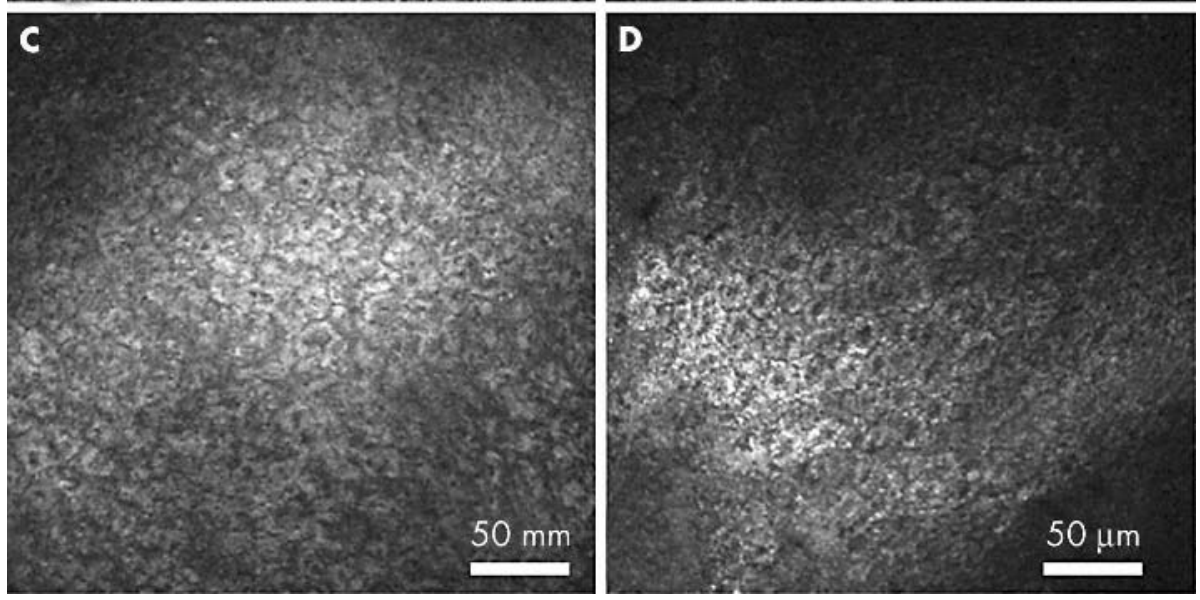

different tissue layers again on the way to the detector. In addition to light intensity loss by virtue of these processes, amplification could occur as a result of the forward scattering of local structures. The calculated scatter intensity of small organelles is about 5000 times higher in the forward direction of the light beam than backwards. ${ }^{10}$

Confocal imaging of the cornea in vivo revealed obvious differences in contrast of similar cellular structures. ${ }^{5}$ Cell membranes of the epithelium are illuminated more brightly than equivalent endothelial membranes. Conditions of the cytoplasm are reversed. The refractive index differs for various cellular structures and averages-for extracellular fluid $\eta=1.35-1.36$, for cytoplasm $\eta=1.36-1.375$, for nuclei $\eta=1.38-1.41$, for mitochondria and organelles $\eta=1.38$ 1.41, for dried protein $\eta=1.58$, for cell membrane $\eta=1.47$, and for lipid $\eta=1.48{ }^{11-16}$ Endothelial cells border the anterior chamber with a conspicuously lower refractive index of aqueous humour of $\eta=1.336,{ }^{8}$ whereas epithelial cells lie embedded in layers of similar refractive conditions at $\eta>1.35$. Our study showed that variation of the refractive index from 1.337-1.47 of the anterior chamber produced no differences in image contrast of endothelial cell structures. The endothelium showed for all cases dark cell borders and bright cytoplasm. Hence, the location of the endothelial layer at the interface to aqueous humour was not the source of varying optical behaviour of epithelial and endothelial cells because of higher refraction index differences.

These differences are also not explainable by loss of intensity, as the effect of overlying structures, if the light goes back from the endothelium, through about 500-600 $\mu \mathrm{m}$ broad cellular structures to the detector. The imaging of a second epithelial cell layer underlying the first corneal endothelium revealed in fact lower image quality as a result of experimental arrangement and procedure, but bright contrasted cell membranes and bright nuclei were visible during our experiment.

There are some morphological differences between endothelial and epithelial cells found by our trans-electron microscopic investigations. The cell membranes of epithelium contained many desmosomes and showed deep interdigitations. In contrast, desmosomes were lacking in endothelium, and cell membranes were smooth. Cell membranes consist of lipids. The membrane interdigitations lead to an abiding appearance of media interfaces with refractive index for lipid of $\eta=1.48$ and for cytoplasm of $\eta=1.48$. Reflection occurs at each interfacing media. For the protein rich desmosomes a value of $\eta>1.5$ could be assumed, given that dried protein has a refractive index of $\eta=1.58 .^{15}$ Therefore, the high concentration of desmosomes could be the source for cell membrane brightness in epithelial images.

Our ultrastructural investigations revealed large numbers of mitochondria in endothelial cells, whereas the number of organelles in epithelial cells was much lower. The effects of various organelles and size of nucleus on the far field scattering patterns of cells were examined with a mathematical model, the finite difference time domain (FDTD) method..$^{10}$ The scattering patterns were computed for cells containing different organelles to determine the effect of each cell component on the total amount of scatter. Scattering functions of two cells with diameters of $11 \mu \mathrm{m}$ were calculated. In the first case the cell contained only a nucleus, membrane, and cytoplasm, in the second case small organelles have been added to the cell. The scattering from a cell was highly peaked in the forward direction. ${ }^{10}$ Small 

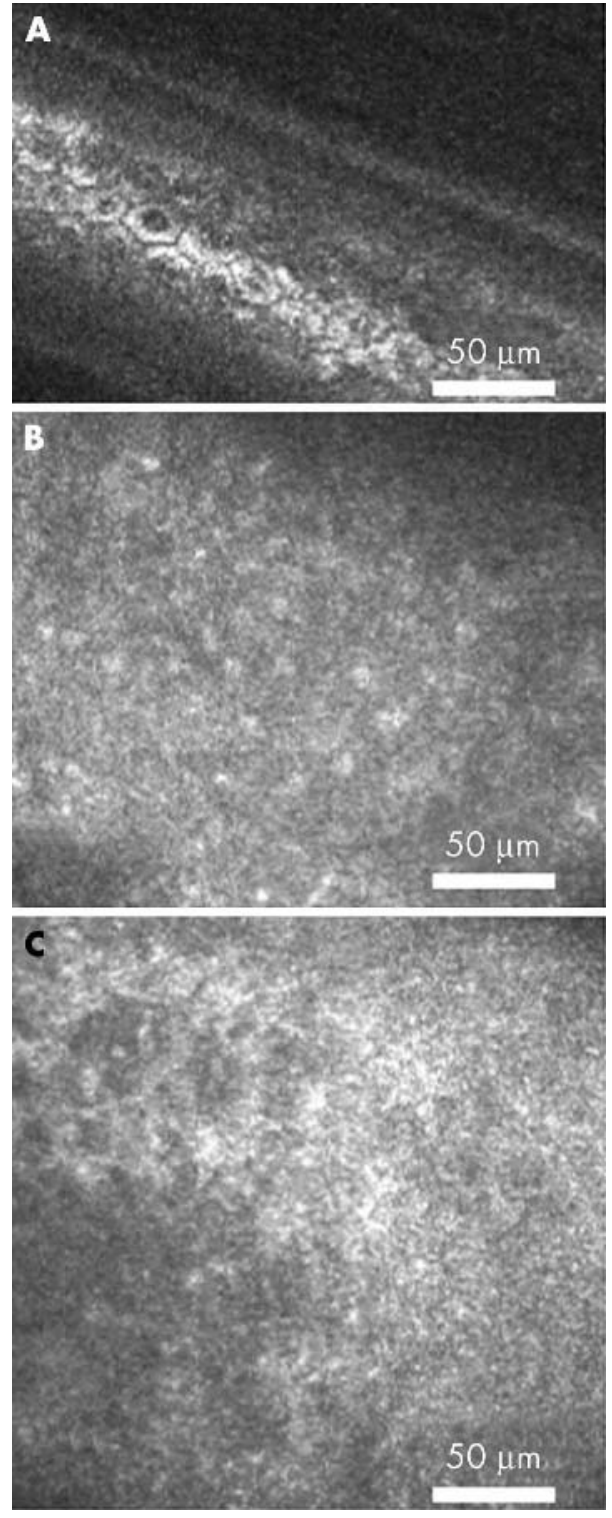

Figure 2 Confocal images of two stacker domestic pig corneas. (A) Endothelium of specimen 1 lying above specimen 2. (B) Superficial cells of specimen 2. (C) Wing cells of specimen 2. Magnification $\times 240$

organelles can have a large effect on the scattering pattern. The total amount of scattered light was 1.7 times greater for the cell with small organelles than for the same cell without the organelles. ${ }^{10}$ This predicts that the small organelles can contribute significantly to the total scattering. Cells containing large nuclei (diameter $6 \mu \mathrm{m}$ ) cause increasing amounts of scattering relative to cells with smaller nuclei (diameter $3 \mu \mathrm{m})$. In the absence of a nucleus, the amplitude of the scattering pattern decreased further. ${ }^{10}$

Our investigations were performed on pig eyes ex vivo. In contrast, this confocal system is applicable to in vivo diagnosis of the human cornea. The experimental use of human eyes is not practicable in vivo and has ethical practical difficulties ex vivo. Therefore, domestic pig eyes, with comparable corneal morphology, are a good study object. However, tissues of ex vivo objects undergo several processes such as cell hydration, and this may change their optical behaviour. The extent of this problem is hard to ascertain. Our confocal observations of pig eyes ex vivo in sequences over a 24 hour period revealed corneal thickening on account

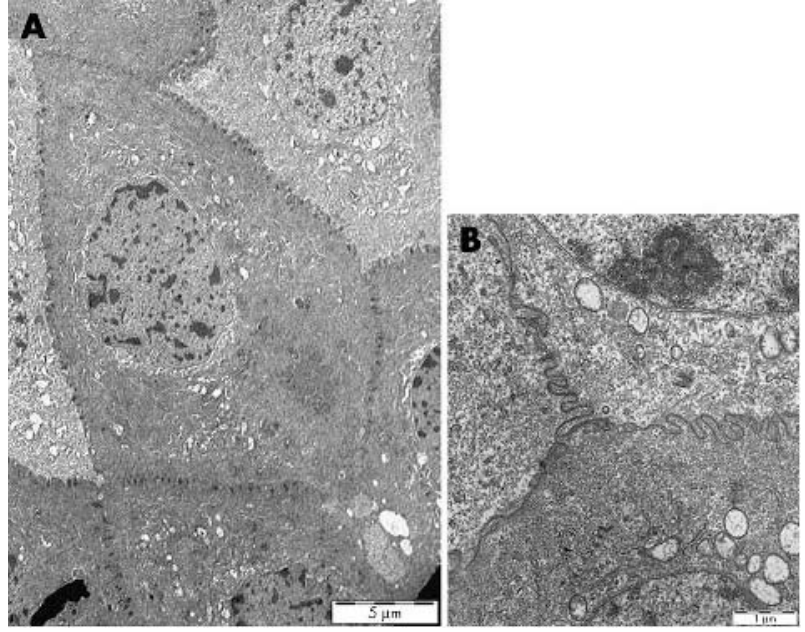

Figure 3 Ultrathin sections of epithelial cells of domestic pig eyes. Magnification $\times 1500$.

of stromal hydration, but the optical behaviour of cells remained similar.

By means of in vivo confocal microscopy, many corneal diseases have been described, as changes in the physiological structure of the tissue produce changes in the contrast of obtained images. ${ }^{5}$ However, the ability to differentiate between normal and abnormal tissue depends on the ability to interpret the source of reflected light within the sample. Our study contributes to the clinical application of the Rostock cornea module and to confocal laser microscopy in general. Observed variations of optical behaviour of corneal endothelium are a result of cell physiology, cell structure, and cell ingredients in the first place. Comparative studies including confocal microscopy and conventional microscopic histology are necessary for further understanding the sources of image contrast.

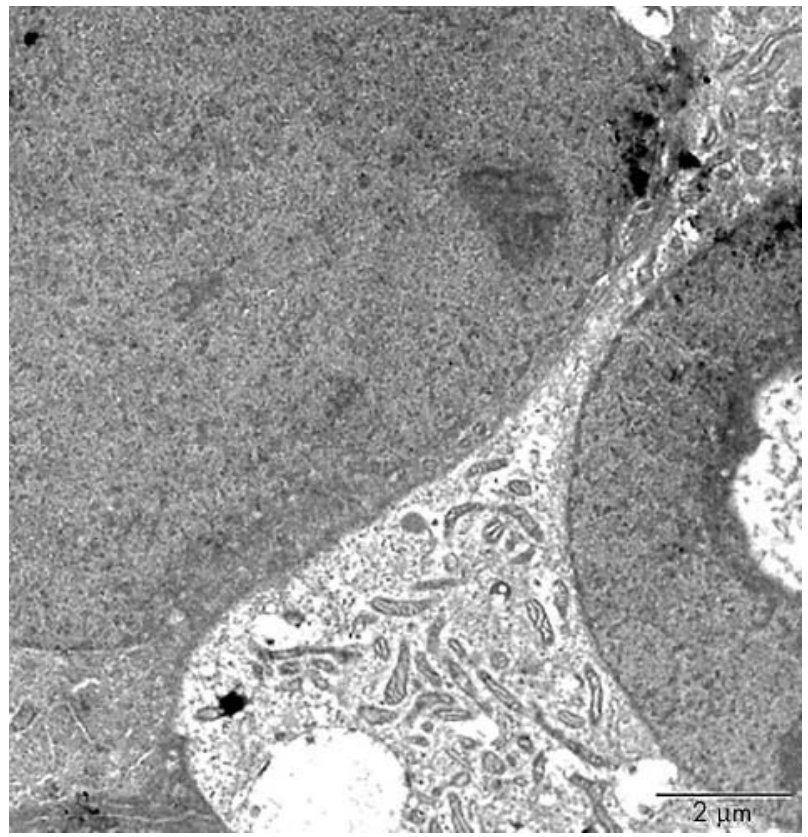

Figure 4 Ultrathin section of endothelial cells of domestic pig showing smooth cell membrane, numberous mitochondria, and homogeneous nuclei. Magnification $\times 12000$. 


\section{ACKNOWLEDGEMENTS}

The authors thank Professor Jonas of the Institute of Pathology at the University of Rostock for use of the electron microscope, and we are grateful to G Fulda for technical assistance. Many thanks to Angus, a native English speaker from Cambridge, for linguistic editing of the manuscript.

\section{Authors' affiliations}

R Bochert, A Zhivov, R Kraak, J Stave, R F Guthoff, Department of Ophthalmology, University of Rostock, Doberaner Strasse 140, 18055 Rostock, Germany

\section{REFERENCES}

1 Calvillo MP, McLaren JW, Hodge DO, et al. Corneal reinnervation after LASIK: prospective 3-year longitudinal study. Invest Ophthalmol Vis Sci 2004;45:3991-6.

2 Nakano E, Oliveira M, Portellinha W, et al. Confocal microscopy in early diagnosis of Acanthamoeba keratitis. J Refract Surg 2004;20(Suppl):737-40.

3 Patel S, McLaren J, Hodge D, et al. Normal human keratocyte density and corneal thickness measurement by using confocal microscopy in vivo. Invest Ophthalmol Vis Sci 2001;42:333-9.

4 Guell JL, Velasco F, Guerrero E, et al. Confocal microscopy of corneas with an intracorneal lens for hyperopia. J Refract Surg 2004;20:778-82.

5 Mastropasqua L, Nubile M. Confocal microscopy of the cornea. Thorofare, NJ: Slack, 2002.
6 Cheng PC, Kriete A. Image contrast in confocal light microscopy. In: Pawley JB, eds. Handbook of confocal microscopy. New York: Plenum Press, 1995:281-310.

7 Smithpeter C, Dunn A, Drezek R, et al. Near real time confocal microscopy of cultured amelanotic cells: sources of signal, contrast agents and limits of contrast. J Biomed Opt 1998;3:429-36.

8 Laing RA, Sandstrom MM, Leibowitz HM. Clinical specular microscopy. II. Qualitative evaluation of corneal endothelial photomicrographs. Arch Ophthalmology 1979:97:1720-25.

9 Stave J, Zinser G, Grummer G, et al. Modified Heidelberg retinal tomograph HRT. Initial results of in vivo presentation of corneal structures. Ophthalmologe 2002;99:276-80

10 Dunn A, Smithpeter C, Welch AJ, et al. Finite-difference time-domain simulation of light scattering from single cells. J Biomed Opt 1997:2:262-6.

11 Maier J, Walker S, Fantini S, et al. Possible correlation between blood glucose concentration and the reduced scattering coefficient of tissues in the near infrared. Opt Lett 1994; 19:2062-4

12 Liu H, Beauvoit B, Kimura M, et al. Dependence of tissue optical properties on solute-induced changes in refractive index and osmolarity. J Biomed Opt 1996;1:200-11.

13 Brunsting A, Mullaney P. Differential light scattering from spherical mammalian cells. Biophys J 1974;14:439-53.

14 Beuthan J, Minet O, Helfman J, et al. The spatial variation of the refractive index in biological cells. Phys Med Biol 1996;41:369-82.

15 Barer R, Joseph S. Refractometry of living cells. Quart J Microscop Sci 1954;95:399-423.

16 Maier J, Walker S, Fantini S, et al. Possible correlation between blood glucose concentration and the reduced scattering coefficient of tissues in the near infrared. Opt Lett 1954;19:2062-4. 